\title{
Clima organizacional sob a perspectiva dos gestores de Instituições Federais de Ensino: uma investigação multivariada em Belém, Pará, Brasil.
}

\section{Organizational Climate Under the Perspective of Managers of Federal Education Institutions: a multivariate research in Belém, Pará, Brazil.}

\author{
CARLOS ANDRÉ CORRÊA DE MATTOS*
}

\section{RESUMO}

O objetivo deste estudo foi avaliar o clima organizacional segundo a percepção de gestores de Instituições Federais de Ensino Superior. A metodologia foi exploratória e descritiva, realizada na forma de levantamento, com amostragem não probabilística por acessibilidade. A amostra contou com 74 servidores públicos em cargo de gestão e o tratamento de dados foi quantitativo. A análise fatorial revelou três fatores, que explicaram $71,67 \%$ da variância dos dados e foram denominados como: Imagem e Desempenho Organizacional (32,43\%), Cooperação e Integração (22,69\%) e Carreira e Oportunidades (16,60\%). A análise de agrupamentos classificou os entrevistados em quatro grupos, diferenciados principalmente pela Cooperação e Integração. Os

* Universidade Federal do Pará (UFPa). Doutor em Ciências Agrárias (2012) pela Universidade Federal Rural da Amazônia (UFRA) e Mestre em Gestão e Desenvolvimento Regional (2008) pela Universidade de Taubaté (Unitau). Cursou MBA em Gestão Empresarial (2004) e Gerenciamento de Projetos (2005) pela Fundação Getúlio Vargas (FGV). Bacharel em Administração (2002) pela Universidade da Amazônia (Unama), na trajetória profissional atuou em organizações públicas e privadas. Dentre as áreas de concentração, destacam-se: Gestão de Organizações Públicas, Competitividade, Agronegócio e Organização Agroindustrial. Atualmente é professor da Faculdade de Administração (FAAD) e do Programa de Mestrado em Gestão Pública para o Desenvolvimento (PPGGPD) da Universidade Federal do Pará (UFPa). E-mail: carlosacmattos@hotmail.com 
grupos tiveram diferentes avaliações do ambiente organizacional, contudo $34 \%$ dos entrevistados avaliaram o clima como favorável nos três fatores. O estudo permitiu concluir que os gestores analisam o clima sob uma perspectiva estratégica, confirmando, em certa medida, a visão diferenciada desses profissionais o que justifica o destaque dado aos gestores nas avaliações de clima organizacional.

Palavras-chave: Ambiente organizacional; análise fatorial exploratória; análise de agrupamentos; escores fatoriais; universidades.

\section{Abstract}

This study aimed to evaluate the organizational climate under the managers' perception of Federal Institutions of Higher Education. The methodology was exploratory and descriptive, accomplished in the form of data survey with non-probabilistic sampling by accessibility. The sample was composed by 74 civil servants in charge of management, and data treatment was quantitative. Factorial analysis showed the presence of three factors, which explained $71.67 \%$ of the data variance and were denominated as Image and Organizational Performance (32.43\%), Cooperation and Integration $(22.69 \%)$, and Professional Career and Opportunities $(16.60 \%)$. y cluster analysis, the interviewees were classified into four groups differentiated mainly by Cooperation. The groups had different evaluations about the organizational climate; however, $34 \%$ of the interviewees evaluated climate as favorable in all three factors. The study allowed to conclude that managers analyze the climate under a strategic perspective, ratifying, to a certain extent, the differentiated view of these professionals, which justifies the emphasis given to the managers in the evaluations of organizational climate.

Keywords: Organizational environment; exploratory factorial analysis; clusters analysis; factorial scores; universities.

\section{INTRODUÇÃo}

As constantes alterações nas práticas gerenciais, intensificadas pelo uso de tecnologias e pela crescente preocupação com a competitividade, desencadearam mudanças que passaram a exigir maior participação do capital humano nas organizações. O trabalho, por muito tempo tido como uma atividade penosa, assumiu 
novos contornos e passou a ser percebido como uma possível fonte de diversão e prazer (SCHAUFELI; DIJKSTRA; VAZQUEZ, 2013). A preocupação com a satisfação com o trabalho (BARBOSA et al., 2016; KJELDSEN; HANSEN, 2016; HORA; RIBAS JÚNIOR; SOUZA, 2018), o reconhecimento profissional (BENDASSOLLI, 2012; SORATTO et al., 2017), assim como as ações de treinamento (ARAÚJO; ABBAD; FREITAS, 2017; MAJEED; SHAKEEL, 2017), além do acompanhamento da qualidade de vida no trabalho (TONGO, 2015; MATTOS et al., 2017), entre outros estudos relativos ao ambiente organizacional, acabaram por colocar os trabalhadores em uma nova posição no horizonte das organizações modernas, tendo isso ocorrido tanto na iniciativa privada, quanto, mais recentemente, no setor público.

Nesse contexto, se insere a preocupação com o clima organizacional, que conforme Litwin e Stringer (1968), pode ser conceituado como um conjunto de propriedades, presentes no ambiente de trabalho, capazes de influenciar no comportamento dos trabalhadores e que podem ser mensuradas, desde que sejam utilizados procedimentos de investigação adequados. Um bom clima organizacional pode ser considerado um estímulo à motivação (BAHRAMI et al., 2014; RUSU; AVASILCAI, 2014), à produtividade, à busca pela qualidade, exercendo, por suas características, forte influência, tanto na satisfação com o trabalho em si, quanto no comprometimento com a organização, aspectos que impactam na performance organizacional sob diversas perspectivas, inclusive a financeira (JAHANI; YAMIN FIROOZ; SIAMIAN, 2015).

Notoriamente, o desempenho dos trabalhadores é influenciado por um amplo conjunto de aspectos, que incluem desde a estrutura da organização, até as formas como as tarefas são distribuídas, o trabalho reconhecido e como a organização apoia a realização das tarefas. Esses mecanismos são alguns dos elementos do clima organizacional, conceituado por Coda (1998, p. 6) como "uma medida de percepção que os empregados têm sobre o grau de satisfação em relação a determinadas características do ambiente de trabalho da organização onde atuam”. Assim, o clima organizacional expressa um amplo conjunto de "conceitos e sentimentos que as pessoas partilham a respeito da organização e que afetam, de maneira 
positiva ou negativa, sua satisfação e motivação para o trabalho" (MAXIMILIANO, 2012, p. 107).

A utilização das pesquisas de clima possibilita obter um diagnóstico amplo e acurado dos principais problemas e virtudes do ambiente de trabalho. Assim, é possível revelar quais necessidades dos trabalhadores estão sendo atendidas e quais não estão. Nesse contexto, as pesquisas de clima possibilitam diagnósticos sob medida, adaptáveis para as realidades de cada organização (KUBO et al., 2015). Com isso, além de trazer elementos que contribuem para a impetração da atmosfera organizacional, as pesquisas de clima revelam aspectos do ambiente de trabalho que são valorizados pelos trabalhadores.

As pesquisas de clima organizacional são ferramentas poderosas para fundamentar a escolha de políticas e estratégias capazes de impulsionar a melhoria do ambiente de trabalho e, por conseguinte, do desempenho individual e organizacional (STRINGER, 2002), tendo sido iniciada sua utilização na administração privada na década de 1960 (FOREHAND; GILMER, 1964). Na administração pública, as pesquisas de clima organizacional ganharam projeção com a valorização do trabalho dos servidores públicos (MÓL et al., 2010; FRANÇA; MATTOS, 2017), que passaram a exercer atribuições mais complexas com o advento do gerencialismo (SILVESTRE, 2010).

O clima organizacional é fortemente influenciado pela atuação dos gestores. A relação estabelecida entre os superiores hierárquicos e seus subordinados é considerada um dos principais influenciadores na formação do clima organizacional (HOLOWAY, 2012), a atuação dos gestores está presente como uma dimensão de avaliação em diversos modelos que mensuram o clima organizacional (CODA, 1998; KOLB et al., 1978; LUCHESI; CRESPI; CAMARGO, 2011; LUZ, 2012). Contudo, os gestores, lideranças ou chefias, como são denominados pela maioria dos modelos, ou ainda os white-collars workers, como são designados em estudos internacionais (BRAND-LABUSCHAGNE et al, 2012; HU, KAPLAN, DALAL, 2010), assumem dupla perspectiva na compreensão do clima. Se a ação dos gestores, por um lado, é considerada uma variável independente por sua força na formação do clima organizacional, por outro, estes gestores não estão imunes ao ambiente 
da organização e, em certa medida, serão influenciados por ele, constituindo, assim, uma avaliação própria do clima organizacional.

Desta forma, ao considerar a perspectiva dos gestores, esse estudo tem por objetivo analisar o clima organizacional de instituições federais de ensino na cidade de Belém do Pará, Brasil, segundo a percepção desses profissionais e, assim, responder ao questionamento: quais fatores influenciam na avaliação do clima organizacional, segundo a percepção dos gestores de instituições federais de ensino superior na cidade de Belém do Pará, Brasil? Para tanto, foram entrevistados servidores públicos dessas instituições que ocupavam cargos de gestão. O caráter inovador desse estudo está em concentrar a investigação nos gestores e, assim, expor a percepção do clima organizacional sob a perspectiva desses profissionais.

\section{REVISÃo DE LITERATURA}

\subsection{Clima organizacional: conceituação, dimensões e pesquisa}

O clima organizacional pode ser conceituado como a percepção compartilhada dos trabalhadores do seu ambiente de trabalho, afetando, essa percepção, tanto o indivíduo em si, quanto o grupo e podendo se refletir em uma parte da organização, ou em toda a organização. Considerado como um constructo capaz de exercer forte influência no comportamento humano (SHIM, 2010; D'ALLEO; SANTANGELO, 2011; MARTELO; CLARO; VIEIRA, 2016), para Luz (2012, p. 6), “o clima organizacional é reflexo do estado de ânimo ou do grau de satisfação dos funcionários de uma empresa, num dado momento".

Laner e Cruz Júnior (2004, p. 55) complementam e conceituam clima como "um conceito psicológico que se refere às percepções molares desenvolvidas pelas pessoas em relação ao seu ambiente de trabalho". Desta forma, observa-se que o clima organizacional é essencialmente uma percepção do ambiente interno da organização, compartilhada entre os trabalhadores, com características circundantes e momentâneas, que se baseia em padrões que ocorrem no contexto específico das experiências organizacionais, podendo receber influências transitórias como aquelas decorrentes de relações momentâneas entre os trabalhadores ou entre eles e a organização. 
O clima organizacional, em uma perspectiva ampla, envolve diversas dimensões que se estendem conforme os diferentes modelos de avaliação, desde aspectos imateriais como treinamentos e calor humano até às condições físicas do local de trabalho.

A avaliação do clima organizacional pode ser feita sob a forma de pesquisa, necessitando, para tanto, de um instrumento capaz de mensurar a percepção do ambiente organizacional. Os gestores podem utilizar as pesquisas de clima para conhecer e, se necessário, melhorar as condições de trabalho nos aspectos que podem ser controlados pela organização. É possível afirmar que o clima organizacional está em constante mudança, sendo um fenômeno temporal, influenciado pelos parâmetros de importância de cada trabalhador, assim as pesquisas refletem essencialmente um momento da organização (LUZ, 2012). As pesquisas de clima organizacional são também um canal de comunicação que liga a organização a suas equipes de trabalho, de modo a manter um feedback se possível regular e adequado à realidade da organização, caracterizado pela opinião que os trabalhadores fazem da realidade organizacional.

Desta forma, as pesquisas proporcionam uma análise conjunta do estado de satisfação ou não com relação a diversos aspectos da organização (CODA, 1998), com o objetivo fornecer informações que possam melhorar a convivência entre a organização e o indivíduo. Assim, a gestão do clima organizacional tem a propriedade de facilitar a execução das atividades diárias ao contribuir para melhorar as relações que ocorrem na organização, ao servir de suporte para a criação de estratégias que visem a adequação da estrutura e dos termos de convivência entre as pessoas (PERREIRA, VIDAL, 2011; SALGADO; AIRES; ARAUJO, 2013). Como destacado, o capital humano progressivamente assumiu maior evidência para o sucesso organizacional, e a combinação entre os interesses pessoais e os organizacionais depende, em grande parte, da intensidade do comprometimento dos trabalhadores, seja no âmbito de organizações pública ou privadas, e as pesquisas de clima são um dos elementos necessários para a gestão do capital humano, uma vez que pode contribuir para a construção de um ambiente de trabalho que favoreça a retenção de talentos (ABREU et al., 2013; FRANCHI; ARENHARDT, 2014). 
A gestão do clima organizacional não compete apenas aos profissionais de recursos humanos, mesmo que eles compartilhem as responsabilidades pela seleção das políticas de gestão de pessoas e assumam a liderança nas pesquisas de clima organizacional. Perreira e Vidal (2011) destacam que a estruturação de um bom clima organizacional é dever de todos os setores da organização que tenham pessoas alocadas para uma finalidade em comum. Esse caráter localizado possibilita a presença de diferentes tipos de clima em diferentes setores ou departamentos organizacionais, aspecto que aumenta a complexidade das decisões nas escolhas de políticas de gestão do clima e desafiam os gestores quanto as estratégias mais adequadas a seguir.

A formação de um clima organizacional saudável é, portanto, uma tarefa comum a toda organização, que se materializa ao considerar as necessidades, percepções, sentimentos e expectativas dos trabalhadores, quando são transformados em políticas organizacionais. As pesquisas de clima aproximam a organização internamente e podem reforçar laços de compromissos. Com isso, contribuem com as interações que possibilitam resultados positivos, tanto para os trabalhadores, quanto para as organizações, constituindo-se nos resultados e no bom funcionamento organizacional (ABREU et al., 2013).

Ao diagnosticar o clima organizacional e atuar para melhorar o ambiente laboral a organização contribui para melhorar sua imagem no mercado. Assim, o clima assume uma dimensão que excede os limites internos e se reflete nos tipos de profissionais que a organização consegue atrair (LANER, CRUZ JÚNIOR, 2004). De acordo com Luz (2012), ao mensurar o clima organizacional, é possível detectá-lo como bom, prejudicado ou ruim e, segundo diferentes dimensões organizacionais, como, por exemplo, instalações, treinamento, reconhecimento pelo trabalho realizado, calor nas relações pessoais, satisfação com o trabalho, salários, benefícios, entre outros. Já para Bispo (2006), as dimensões que influenciam a mensuração do clima organizacional abrangem também outros fatores, externos à organização, como, por exemplo, convivência familiar, férias, lazer, time de futebol, entre outros.

Com o passar dos anos, a quantidade de dimensões, estudadas para identificar as condições do clima organizacional, se multiplica- 
ram, levando à imprecisão e a um lento progresso teórico (PATTERSON et al., 2005). Por outro lado, como destacam Kubo et al. (2015), a diversidade de dimensões se reflete na flexibilidade da análise. Essa condição que, se por um lado, denuncia a falta de suporte teórico mais delimitado e bem fundamentado para o clima organizacional, por outro, justifica a presença das pesquisas de clima na fronteira das investigações do ambiente organizacional, uma vez que a ausência de dimensões rígidas contribui para a adequação das pesquisas às peculiaridades de cada organização, promovendo diagnósticos sob medida, condição que garantiu a longevidade das pesquisas de clima organizacional ao longo do tempo (KUBO et al., 2015).

Coda (1998) reforça e recomenda que a investigação do clima organizacional deve iniciar pelas definições operacionais e seleção das dimensões que serão investigadas. Ramos Filho (2008) reconhece as incontáveis possibilidades de dimensões para pesquisa de clima organizacional e Patterson et al. (2005) e Kubo et al. (2015) recomendam que as dimensões do clima organizacional devem se diferenciar de acordo com a proposta de investigação e os critérios de interesse da organização, considerando preferencialmente dimensões gerais e mais específicas de cada organização, incluindo aspectos afetivos.

No caso das instituições de ensino superior, Luchesi, Crespi e Camargo (2011) recomendam, para pesquisas de clima, dimensões como imagem institucional, políticas institucionais, sistema de assistência e benefícios, estrutura organizacional, organização e condições de trabalho, relacionamento interpessoal, comportamento das chefias, satisfação pessoal, planejamento institucional, processo decisório, autonomia universitária e avaliação institucional, expandindo assim a análise da estrutura do clima na organização para diversas dimensões de análise. Em contrapartida, Abreu et al. (2013), ao averiguarem um hospital universitário, utilizaram dimensões como comunicação, contexto social, situação financeira e imagem institucional.

Lim e Eo (2014), por outro lado, investigaram o clima organizacional de acordo com aspectos relacionados com a comunicação, a percepção das políticas organizacionais, os níveis de eficácia coletiva dos professores, o esgotamento profissional (burnout) e os procedimentos organizacionais. Ramos Filho (2008) adverte, que, pelas múltiplas possibilidades de dimensões do clima organizacional, há 
atualmente vários modelos disponíveis para estudá-lo, inexistindo um modelo universal com possibilidade de aplicação em quaisquer organizações, tornando incontáveis as possibilidades de dimensões para pesquisa de clima organizacional contemporaneamente.

\subsection{Clima organizacional em instituições de ensino superior}

Instituições de Ensino Superior são organizações naturalmente complexas, uma vez que reúnem, simultaneamente, atividades como ensino, pesquisa e extensão. Essa complexidade se reflete no objetivo de produzir e transmitir conhecimento para formar cidadãos e melhorar a sociedade. Nesse contexto, essas organizações mostram particularidades tanto de ordem estrutural, quanto de funcionamento, que as distinguem das demais organizações (TACHIZAWA; ANDRADE, 2008; CAMPOS, 2002; VIEIRA, 2007). Destaca-se também que a valorização da educação na sociedade moderna aumentou o prestígio e a necessidade de gerenciar de forma mais eficiente as organizações de ensino (ARABACI, 2010).

Arabaci (2010) destaca que as organizações devem promover comportamentos adequados em seus trabalhadores e, assim, dirigir ações que conduzam seu pessoal rumo a maneiras mais eficientes e produtivas de trabalho. Desta forma, em se tratando de instituições de educação, observa-se que esse tipo de organização reúne aspectos muito diferentes entre a atuação do pessoal acadêmico e administrativo, especialmente quanto ao formalismo e à estruturação das tarefas. Isso resulta em diferentes percepções quanto ao clima, que pode ser considerado sob diferentes feitios, como, por exemplo, aberto, restritivo, sincero, reservado, entre outros. Compreendê-lo nessas instituições pode contribuir para a realização individual dos trabalhadores e para melhorar a qualidade do ensino.

A inserção social das instituições de ensino promove singularidades que as diferenciam de outras organizações, uma vez que enquanto as organizações industriais e comerciais visam a resultados comprovados pela produção de bens e entrega de serviços. As instituições de ensino conservam seu foco na formação de seres humanos para conviver em sociedade, com direitos, deveres, e no caso específico das instituições de ensino superior, com especificidades de ordem profissional e ética, aspecto que aumenta sua 
complexidade (TACHIZAWA; ANDRADE, 2008). Destaca-se assim uma responsabilidade social das instituições de ensino superior ao buscar a formação de cidadãos capazes de promover transformações na sociedade.

Sendo uma organização com características muito específicas, as instituições de ensino revelam uma cultura definida, em grande parte, por suas tarefas e objetivos. Obviamente, elas se subordinam a um conjunto de normas, regras e estatutos, muitos deles com elevada formalidade e rigor, que fazem com que essas organizações sejam comumente apegadas a tradições e a aspectos burocráticos que, por conseguinte, se refletem na cultura e no clima organizacional (CAMPOS, 2002).

Na década de 1980, Moran e Volkwein (1992) desenvolveram pesquisas com foco no clima organizacional em instituições de ensino, tendo esses estudos sido considerados como pioneiros na área. No Brasil, entre os anos de 1995 a 2002, as pesquisas de Rizzatti (2002) se destacaram ao apresentar doze categorias de análise de clima organizacional em universidades federais brasileiras e ao gerar diagnósticos capazes de aperfeiçoar diversos processos das instituições estudadas (SANTOS; VÁSQUEZ, 2012).

Moro et al. (2012) avaliaram fatores que interferem no clima organizacional de servidores técnico-administrativos de uma instituição pública de ensino. Os dados foram obtidos com a utilização de questionários baseados no modelo de Luz (2012). Os resultados revelaram elevado comprometimento e satisfação com o clima organizacional. Contudo, havia baixo reconhecimento profissional, dificuldades para a progressão, e a remuneração foi considerada insatisfatória. Esses aspectos recomendaram a necessidade de pesquisas futuras de clima, especialmente pelas características das organizações públicas de ensino superior e pelas expectativas da sociedade quanto a essas organizações.

Dentre as pesquisas de clima organizacional realizadas em instituições de ensino Mejías et al. (2006) desenvolveram um instrumento, tendo como base as propostas de Litwin e Stringer (1968). Este instrumento foi desenvolvido com o propósito de gerenciar o ambiente de trabalho de forma interdisciplinar, além de mensurar o clima organizacional universitário na perspectiva da gestão da qua- 
lidade. Segundo os autores, o modelo se mostrou apropriado para a avaliação do clima organizacional em instituições de ensino superior.

Concentrando-se nos professores, Cantos, Silva e Nunes (2005), ao estudar as causas do estresse e o impacto das condições de trabalho de uma instituição pública de ensino superior no Brasil, constataram que o excesso de atividades, como reuniões, responsabilidades diversificadas, falta de tempo para realizar todas as tarefas requeridas, causa frustração, fadiga, desentendimentos e desarmonia entre os professores. Assim, as pesquisas de clima foram consideradas necessárias para subsidiar medidas mitigadoras de impactos negativos na saúde dos professores e na qualidade dos serviços na instituição.

Luchesi, Crespi e Camargo (2011) complementam ao reforçar que as instituições de ensino superior têm o compromisso de fomentar o questionamento e formar cidadãos capazes de impulsionar melhorias na sociedade. Porém, como em toda organização, a qualidade de seus serviços depende fortemente de seus profissionais, que, nesse caso específico, são os professores e os técnico-administrativos. Desta forma, é essencial analisar a motivação e a percepção destes profissionais quanto à qualidade de seu ambiente de trabalho, sendo as pesquisas de clima um meio eficaz para possibilitar essa análise.

Santos e Vasquez (2012) destacam que as instituições de ensino superior podem contribuir expressivamente para o desenvolvimento sustentável. Desta forma, os esforços empreendidos para melhorar o ambiente de trabalho dessas instituições se revertem em externalidades positivas, visto que essas instituições impulsionarem o progresso e a transmissão de conhecimento por meio das pesquisas, inovações e da formação profissional, garantindo o avanço tecnológico que se materializa na melhoria nas condições de vida. Os estudos apresentados possibilitam constatar que as pesquisas de clima organizacional em instituições de ensino contribuem objetivamente para melhorar a administração do sistema educacional, sendo possível corrigir diversas situações prejudiciais ao ambiente de trabalho pela sua utilização, e que as chefias são agentes de destaque na formação desse ambiente. 


\section{Metodologia}

Os procedimentos metodológicos utilizados neste estudo caracterizam uma investigação exploratória feita em forma de levantamento, com amostragem não probabilística por acessibilidade e tratamento de dados quantitativo. Esse tipo de estudo busca uma aproximação inicial do fenômeno para posterior aprofundamento em estudos de caráter descritivo ou explicativos (VERGARA, 2014; MARTINS; TEÓPHILO, 2017). O levantamento, por sua vez, é uma forma de coleta de dados que ocorre no mesmo local do fenômeno.

O universo de pesquisa foi formado por servidores públicos de duas Instituições Federais de Ensino Superior (IFES), a Universidade Federal Rural da Amazônia (UFRA) e o Instituto Federal de Educação, Ciência e Tecnologia do Estado do Pará (IFPA), ambas localizadas na cidade de Belém do Pará, Brasil, que exerciam funções de chefia, portanto, além de receber Funções Gratificadas (FG) e gratificação de Cargo de Direção (CD), apresentavam pelo menos um subordinado por ocasião do levantamento. Esses profissionais foram selecionados por acessibilidade, portanto, convidados a participar da pesquisa. Ao final da coleta de dados, foram obtidos 74 questionários de pesquisa válidos. A técnica de amostragem, por não utilizar critérios probabilísticos na seleção dos entrevistados, não permite a mensuração do erro, nem o cálculo da representatividade, limitando os achados aos entrevistados (MARTINS; TEÓPHILO, 2017).

A investigação assumiu um caráter aplicado, uma vez que se concentrou em aspectos do cotidiano organizacional (VERGARA, 2014). O levantamento foi feito no próprio local de trabalho dos entrevistados e utilizou o questionário como instrumento de pesquisa. O questionário foi composto por duas seções: uma destinada a caracterizar o perfil sociodemográfico dos entrevistados e outra elaborada na forma de assertivas relacionadas com aspectos do clima organizacional, tendo sido reunidas nas dimensões comunicação organizacional, imagem organizacional, cooperação, remuneração e recompensa, satisfação com o trabalho, equidade, participação nas decisões, valorização do servidor e modernidade organizacional. As assertivas tiveram respostas na escala intervalar de Lickert, com cinco opções de respostas: 1 para discordo totalmente, 2 para 
discordo, 3 para não concordo, nem discordo, 4 para concordo e 5 para concordo totalmente.

No tratamento dos dados, foram empregadas técnicas quantitativas, entre elas, estatística descritiva e multivariada. As técnicas descritivas incluíram a distribuição de frequência e as medidas de dispersão e de tendência central. Com relação às técnicas multivariadas, foram selecionadas a análise fatorial exploratória (AFE) e a análise de agrupamentos (AA), selecionadas pela capacidade de sumarizar grande volume de dados em reduzido número de fatores e grupos (HAIR et al., 2009), simplificando a interpretação dos resultados. A análise fatorial exploratória é uma técnica de análise de dados multivariada, capaz de identificar fatores latentes, possibilitando, com isso, criar indicadores ou dimensões de análise com o mínimo de perda de informação possível (FÁVERO et al., 2009). O modelo de análise fatorial (1) pode ser representado, conforme Dillon e Goldstein (1984):

$$
X=\alpha F+\varepsilon,
$$

Em que $X$ é o p-dimensional, vetor transposto das variáveis observáveis, denotado por $X=\left(x_{1}, x_{2} \ldots, x_{p}\right)^{\text {t; }} F$ é o q-dimensional, vetor transposto das variáveis não observáveis ou variáveis latentes, denominadas de "fatores comuns", denotados por $F=\left(f_{1}, f_{2^{\prime}} \ldots, f_{q}\right)$ t, sendo $\mathrm{q}<\mathrm{p}$; $\varepsilon$ é o $\mathrm{p}$-dimensional, vetor transposto de variáveis aleatórias ou fatores únicos, $\varepsilon=\left(\mathrm{e}_{1}, \mathrm{e}_{2}, \ldots, \mathrm{e}_{\mathrm{p}}\right)^{\mathrm{t}}$; e $\alpha$ é a matriz $(\mathrm{p}, \mathrm{q})$ de constantes desconhecidas, identificadas como "cargas fatoriais".

Os fatores após extraídos foram verificados quanto à consistência interna pelo coeficiente Alpha de Cronbach (2). Costa (2011) destaca que esse coeficiente é o mais utilizado nas pesquisas por sua elevada capacidade de mensurar a fidedignidade dos dados. Desta forma, ele possibilita averiguar se as respostas se repetiriam caso o mesmo instrumento fosse aplicado novamente na mesma amostra. Apesar de não existir um padrão para a interpretação do coeficiente, ele é considerado satisfatório quando é superior a 0,600 (HAIR et al., 2009).

$\alpha=\frac{\mathrm{k}}{\mathrm{k}-\mathbf{1}}\left(\mathbf{1}-\frac{\sum_{i=1}^{\mathrm{k}} \sigma_{i}^{2}}{\sigma_{\mathrm{y}}^{2}}\right)$ 
Em que k é o número de itens do questionário; $\sigma_{\mathrm{i}}^{2}$ é a variância do item i; e $\sigma_{\mathrm{y}}^{2}$ é a variância da escala total somada.

Quanto à análise de agrupamentos (clusters analisys), ela é uma técnica de análise de dados multivariada, que depende muito do conhecimento prévio do pesquisador, pois se caracteriza por ser descritiva, ateorética e não inferencial. Essa técnica utiliza algoritmos e se baseia no cálculo da distância geométrica entre as observações para formar agrupamentos. Ela permite formar grupos homogêneos, reunindo observações ou entrevistados com a maior similaridade possível. Ao final da utilização da técnica, os grupos mostram grande similaridade interna e dissimilaridade entre os grupos formados (HAIR et al., 2009).

Neste estudo, a formação dos agrupamentos utilizou procedimento hierárquico aglomerativo, calculado com base na distância euclidiana ao quadrado (3) e o algoritmo de Ward como critério de aglomeração. Segundo esse método, os agrupamentos serão formados pela menor soma dos quadrados, calculada sobre todas as variáveis (FÁVERO et al., 2009). O algoritmo utilizado na formação dos grupos tende a formar agrupamentos semelhantes quanto ao número de integrantes.

$$
\mathrm{d}_{\mathrm{ij}}^{2}=\sum_{\mathrm{k}=1}^{\mathrm{p}}\left(\mathrm{x}_{\mathrm{ik}}-\mathrm{x}_{\mathrm{jk}}\right)^{2}
$$

Em que $\mathrm{d}^{2}{ }_{\mathrm{ij}}$ é $\mathrm{j}$-ésima característica do i-ésimo indivíduo; $\mathrm{x}_{\mathrm{ik}}$

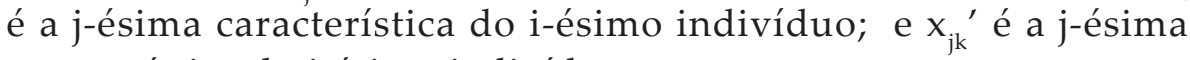
característica do i-ésimo indivíduo.

Na formação dos grupos, foi utilizado o escore fatorial, destaca-se que o escore fatorial é padronizado, portanto, quando calculado para todas as observações, resulta em média zero $(0,00)$ e desvio padrão um $(1,00)$. Desta forma, quanto mais a média e o desvio padrão dos agrupamentos se distanciarem desses valores, maior será a intensidade da percepção favorável do clima organizacional, se assumirem valores positivos, ou insatisfação se negativos. Os escores fatoriais (4), como destacam Hair et al. (2009) e Fávero et al. (2009), são capazes de substituir as variáveis originais em outras análises posteriores e são expressos conforme Mingoti (2007): 


$$
\hat{F}_{i j}=w_{j 1} Z_{1 k}+w_{j 2} Z_{2 k}+\cdots+w_{j p} Z_{p k}
$$

Em que $\left(Z_{1 k} Z_{2 k} \ldots Z_{p k}\right)$, são os valores observados das variáveis padronizadas $Z_{i}$ para o k-ésimo elemento amostral e os coeficientes $W_{\text {ip }} \mathrm{i}=1,2$, ...p são os pesos de ponderação de cada variável $Z_{i}$ no fator $\mathrm{F}_{\mathrm{j}}$.

\section{Apresentação de Resultados}

A amostra foi formada por 74 (100\%) gestores de instituições federais de ensino. Quanto à categoria profissional, na maioria, eram professores, com 38 (51\%) entrevistados posicionados nessa categoria, com idade média de 42 anos ( \pm 9 anos), sendo 41 (55\%) do sexo masculino. Os entrevistados tinham escolaridade elevada: $28(38 \%)$ tinham título de doutorado, 74 (100\%) ocupavam cargos efetivos e tinham em média 12 anos ( \pm 9 anos) de experiência profissional, 48 (65\%) eram casados e 55 (74\%) tinham experiência na iniciativa privada. Os gestores, por ocasião da entrevista, reuniam em média $7( \pm 13)$ servidores sob seu comando, e $57(77 \%)$ indicariam a organização como um bom local para trabalhar. Quanto à renda familiar, $34(46 \%)$ dos entrevistados declararam valores entre 9.989 e 17.433,00 reais por mês.

\subsection{Análise Fatorial Exploratória}

A matriz de dados mostrou boa adequação para utilização da análise fatorial, mesmo considerando a baixa relação entre o número de observações e de variáveis $(1,85)$. A determinante diferente de zero, o teste KMO calculado em 0,861 e o teste de esfericidade de Bartlett, significativo a 1\%, evidenciaram a boa adequação da matriz de dados à técnica da análise fatorial, conforme recomendam Hair et al. (2009), Fávero et al. (2009) e Rodrigues (2009). Atendida a etapa inicial, foi feita a extração dos fatores com a utilização do critério do autovalor, combinado com o método dos componentes principais e rotação ortogonal Varimax. Foram obtidos três fatores, que explicaram $71,67 \%$ da variância dos dados. O coeficiente alpha de Cronbach, com valores acima de 0,730, revelou boa consistência interna, e a comunalidade, superior a 0,681 para todas as variáveis, mostrou que os fatores explicaram a maior parte da variância das variáveis (Tabela 1$)$. 
O primeiro fator reuniu seis variáveis (V14, V17, V18, V16, V15, V02) e captou a atuação da gestão organizacional. O fator sumarizou aspetos que refletem essencialmente a preocupação com a atuação da organização perante a sociedade, aos clientes e demais organizações, por esse motivo, o fator foi denominado como Imagem e Desempenho Organizacional. O segundo fator, com quatro variáveis (V05, V06, V01 e V03), foi denominado como Cooperação e Integração, uma vez que reuniu variáveis que representam um ambiente de trabalho de ajuda mútua e de respeito aos trabalhadores. O terceiro fator, composto por três variáveis (V12, V13, V04), foi denominando como Carreira e Oportunidades, uma vez que captou as formas de avaliação do trabalho, a liberdade para executá-lo e a possibilidade de crescimento na estrutura organizacional.

Tabela 1 - Variáveis, fatores, cargas fatoriais, comunalidade, variância explicada e alpha de Cronbach

\begin{tabular}{|c|c|c|c|c|c|}
\hline \multirow{2}{*}{\multicolumn{2}{|c|}{$\begin{array}{l}\text { Variáveis } \\
1\end{array}$}} & \multicolumn{3}{|c|}{ Fatores } & \multirow{3}{*}{$\begin{array}{l}\text { h2 } \\
0,792\end{array}$} \\
\hline & & \multirow{2}{*}{\begin{tabular}{|l|}
2 \\
0,860
\end{tabular}} & \multirow{2}{*}{\begin{tabular}{|l|}
3 \\
0,052
\end{tabular}} & \multirow[b]{2}{*}{0,220} & \\
\hline $\begin{array}{l}\text { Percebo que a organização em que } \\
\text { trabalho tem boa imagem perante a } \\
\text { sociedade. }\end{array}$ & V14 & & & & \\
\hline $\begin{array}{l}\text { Noto que os usuários estão satisfei- } \\
\text { tos com os serviços oferecidos pela } \\
\text { organização. }\end{array}$ & V17 & 0,856 & 0,184 & $-0,085$ & 0,774 \\
\hline $\begin{array}{l}\text { A organização em que trabalho } \\
\text { aceita que os servidores escolham } \\
\text { as melhores maneiras para realizar } \\
\text { seu trabalho. }\end{array}$ & V18 & 0,815 & 0,240 & 0,230 & 0,776 \\
\hline $\begin{array}{l}\text { Na organização em que trabalho, } \\
\text { há justiça na distribuição das tare- } \\
\text { fas entre os servidores. }\end{array}$ & V16 & 0,803 & 0,131 & 0,301 & 0,752 \\
\hline $\begin{array}{l}\text { A organização que trabalho é res- } \\
\text { peitada pelas outras organizações } \\
\text { federais. }\end{array}$ & V15 & 0,749 & 0,147 & 0,301 & 0,673 \\
\hline $\begin{array}{l}\text { A organização em que trabalho se } \\
\text { preocupa em inovar em formas de } \\
\text { gestão. }\end{array}$ & V02 & 0,739 & 0,267 & 0,175 & 0,648 \\
\hline
\end{tabular}




\begin{tabular}{|c|c|c|c|c|c|}
\hline \multirow{2}{*}{\multicolumn{2}{|c|}{$\begin{array}{l}\text { Variáveis } \\
1\end{array}$}} & \multicolumn{3}{|c|}{ Fatores } & \multirow{3}{*}{$\begin{array}{l}\text { h2 } \\
0,729\end{array}$} \\
\hline & & \multirow{2}{*}{2} & \multirow{2}{*}{$\begin{array}{l}3 \\
0,829\end{array}$} & \multirow[b]{2}{*}{0,076} & \\
\hline $\begin{array}{l}\text { No meu local de trabalho, todos os } \\
\text { servidores são tratados com respei- } \\
\text { to, independentemente do cargo } \\
\text { que ocupam. }\end{array}$ & V05 & & & & \\
\hline $\begin{array}{l}\text { Existe cooperação, mesmo que } \\
\text { informal, entre meus colegas de } \\
\text { trabalho e departamentos da orga- } \\
\text { nização. }\end{array}$ & V06 & 0,302 & 0,777 & $-0,060$ & 0,698 \\
\hline $\begin{array}{l}\text { As chefias ajudam os servidores na } \\
\text { execução das tarefas. }\end{array}$ & V01 & 0,165 & 0,748 & 0,241 & 0,645 \\
\hline $\begin{array}{l}\text { Confio nas informações transmiti- } \\
\text { das pelos meus superiores hierár- } \\
\text { quicos. }\end{array}$ & V03 & 0,035 & 0,694 & 0,441 & 0,677 \\
\hline $\begin{array}{l}\text { O trabalho que realizo é avaliado } \\
\text { de maneira imparcial por meus } \\
\text { superiores hierárquicos. }\end{array}$ & V12 & 0,247 & 0,208 & 0,780 & 0,712 \\
\hline $\begin{array}{l}\text { Percebo que tenho oportunidades } \\
\text { de ser promovido e ascender pro- } \\
\text { fissionalmente }\end{array}$ & V13 & 0,313 & 0,044 & 0,720 & 0,618 \\
\hline $\begin{array}{l}\text { Meus superiores dão liberdade } \\
\text { para os servidores expressarem } \\
\text { livremente suas opiniões quanto à } \\
\text { organização e ao trabalho realiza- } \\
\text { do. }\end{array}$ & V04 & 0,117 & 0,605 & 0,670 & 0,828 \\
\hline Autovalores & & 4,26 & 2,95 & 2,16 & 9,37 \\
\hline Variância Explicada (\%) & & 32,43 & 22,69 & 16,60 & 71,67 \\
\hline Apha de Cronbach & & 0,919 & 0,730 & 0,758 & 0,904 \\
\hline
\end{tabular}

Fonte: Pesquisa de campo.

O fator Imagem e Desempenho Organizacional explicou $32,43 \%$ da variância dos dados e sintetizou uma preocupação típica da atuação gerencial. Nesse sentido, a busca por melhorias na performance da organização não é uma preocupação recente, uma vez que alcançar melhores resultados está entre os principais interesses de gestores desde o início da administração científica. Complemen- 
tarmente, o desempenho organizacional relaciona-se fortemente com a capacidade competitiva (BRANDÃO; BORGES-ANDRADE, GUIMARÃES, 2012) e exprime um compromisso esperado dos ocupantes de cargos estratégicos.

A relação entre clima e desempenho organizacional está presente em diversos estudos (GRIFFITH, 2006; JING; AVERY; BERGSTEINER; 2010; SHANKER et al., 2017) constituindo uma dimensão de avaliação do clima nos modelos de Salgado Neto (2001) e Luz (2012) nos quais é identificado respectivamente como padrões de desempenho e orientação para resultados. A influência do clima no desempenho foi estudada por Stringer (2002) no Modelo de Desempenho Organizacional, segundo o qual a motivação e o desempenho, tanto individual, quanto do grupo, seriam o resultado de um amplo conjunto de forças, internas e externas, que, atuando na cultura e no clima organizacional, seriam capazes de influenciar no desempenho da organização como um todo. Nessa perspectiva, Shanker et al. (2017) reforçam ao afirmarem que, quando as organizações conseguem promover um clima organizacional positivo, é mais provável que elas consigam níveis mais elevados de motivação (RUSU; AVASILCAI, 2014), compromisso e envolvimento dos trabalhadores, o que leva naturalmente a um melhor desempenho (JING; AVERY; BERGSTEINER, 2010).

Cavazotte, Moreno e Turano (2015) complementam e afirmam que, juntamente com os recursos organizacionais, a cultura influencia fortemente no desempenho da organização, pois ela representa um elemento essencial para compreender como serão orientadas as decisões e estabelecidas as prioridades (KOTTER; HESKETT, 2011). Assim, pela proximidade entre cultura e clima organizacional, os esforços para melhorar o clima podem representar uma estratégia para aumentar o compromisso dos trabalhadores com a organização o que se reflete no desempenho e, por conseguinte, na imagem da organização junto aos stakeholders.

Holoway (2012), ao estudar a relação entre clima e liderança, compreende que os trabalhadores são o principal ativo organizacional e que os líderes têm grande influência na percepção, emoções, moral, atitudes e comportamento dos trabalhadores, de tal forma que suas ações têm potencialidade para promover continuamente um 
clima positivo no ambiente de trabalho. Ainda segundo o autor, as lideranças exprimem o que a organização espera dos trabalhadores ao transmitir as expectativas e os valores organizacionais.

Nessa perspectiva, a presença do fator Imagem e Desempenho Organizacional revela que os gestores que participaram da pesquisa são sensíveis à performance da organização ao entenderem que a imagem institucional, a satisfação dos usuários, o respeito conquistado junto a outras organizações, além da liberdade conferida aos trabalhadores para escolher as melhores maneiras para realizar suas tarefas, combinada à inovação em técnicas de gestão, contribuirão para um melhor ambiente de trabalho. Essa compreensão sugere que os gestores reconhecem que a Imagem e Desempenho Organizacional exprimem um indicativo das capacidades da organização, ofuscando a compreensão burocrática da sobreposição do processo sobre o resultado.

O fator Cooperação e Integração, segundo fator identificado na pesquisa, explicou 22,69\% da variância e refletiu questões internas do ambiente de trabalho, pois captou a articulação entre os trabalhadores entre si e entre as unidades de trabalho, mesmo que essa articulação não seja o resultado de uma diretriz organizacional. $\mathrm{O}$ fator reuniu aspectos relativos ao tratamento respeitoso dado aos trabalhadores, à colaboração entre eles e entre as unidades, à solidariedade prestada pelas chefias durante a realização das tarefas e à credibilidade dada às informações recebidas de superiores hierárquicos. Aspecto semelhante foi identificado no modelo de Luz (2012) no qual foi denominado como integração entre departamentos.

A composição das assertivas presentes na formação desse fator sugere que, para os gestores, a autonomia nas equipes e sua integração são aspectos valorizados. Nesse sentido, encontrar um equilíbrio na relação centralização versus delegação é um desafio aos gestores e, dependendo do comportamento de cada um, pode levar à sobrecarga pessoal ou à sobrecarga dos trabalhadores e, como destaca Shim (2015), para ambas as situações, existe um viés gerencial que leva à ineficiência e ao desperdício de recursos.

Landeo e Spier (2015) esclarecem que nas organizações em que ocorrem interações contínuas e de longo prazo entre os trabalhadores surgem nas equipes de trabalho incentivos implícitos que 
promovem a cooperação. Assim, a própria interação entre os pares pode promover sanções que levem a um processo cooperativo ao incentivar os mais resistentes a um maior envolvimento com as tarefas. Os mesmos autores destacam que a complementariedade é a principal razão para o trabalho cooperado e que as organizações devem promover programas de incentivos que possam ser individualizados, uma vez que os programas que recompensam as equipes de forma compartilhada, leva a um comportamento de descompromisso e a uma baixa cooperação. Contudo, desenvolver programas de incentivos tem sido um desafio na administração pública mundial com raros resultados exitosos, como destacam Assis e Reis Neto (2011) e a Organization for Economic Co-operation and Development (OCDE, 2005).

Outro aspecto de destaque é que um processo eficiente de comunicação induz a uma melhor coordenação. Nessa perspectiva, a comunicação, combinada com a interação de longo prazo entre os integrantes das equipes de trabalho, facilita a atuação do gestor, propiciando um mecanismo eficiente de coordenação. Assim, a interação contínua entre os membros da equipe afeta positivamente o retorno do principal, refletindo-se na organização de maneira ampla (LANDEO; SPIER, 2015). Rusu e Avasilcai (2014) seguem a mesma linha ao colocarem em foco que a colaboração influencia positivamente na melhoria de performance, uma vez que os trabalhadores valorizam os comentários positivos de colegas e superiores.

O fator Carreira e Oportunidades, terceiro fator da pesquisa, explicou $16,60 \%$ da variância dos dados e evidenciou a avaliação imparcial pelo trabalho realizado. No mesmo sentido, o fator captou os mecanismos que possibilitam as promoções e a evolução na hierarquia da organização e a liberdade de expressão quanto a organização e as tarefas. A combinação desses elementos constitui um ambiente de trabalho de livre expressão, tendo como bases a justição e a imparcialidade, em que as oportunidades são disponibilizadas com imparcialidade.

Carreira, segundo Bergue (2011, p. 217), reúne um “conjunto de cargos afins, dispostos em posições ordenadas segundo uma trajetória evolutiva crescente de variação e exigências requeridas para 
ascensão". Lu et al. (2015) destacam que, quando os trabalhadores percebem práticas de gestão de recursos humanos concienciosas, diligentes e justas, eles retribuem a organização com atitudes positivas que melhoram o clima organizacional e promovem um desempenho superior.

\subsection{Análise de Agrupamentos}

A análise de agrupamentos foi feita tomando por base o valor dos escores fatoriais calculados pelo método da regressão. Destaca-se que os escores medem posições relativas e, quando calculados para toda a amostra, resultam em média 0,00 (zero) e desvio padrão 1,00 (um). Desta forma, a interpretação dos agrupamentos deve basearse nesses valores. Assim, quanto mais as médias dos agrupamentos se distanciarem de zero, maior será a satisfação ou importância do fator se o sinal for positivo, ou ao contrário, insatisfação ou baixa importância se o sinal assumir valor negativo. Ao final do processo aglomerativo, foram identificados quatro agrupamentos entre os entrevistados, com, respectivamente, 9 (12\%), 25 (34\%), 28 (38\%) e $12(16 \%)$ integrantes.

$\mathrm{Na}$ interpretação dos agrupamentos, as médias dos escores que se posicionaram entre 0,000 e 0,500 foram consideradas baixas; entre 0,501 e 1,000, intermediárias; e acima de 1,001, elevadas. Os agrupamentos foram testados para verificar se estavam corretamente classificados e qual fator diferenciava mais os agrupamentos. Esse procedimento foi feito com o teste não paramétrico de Kruskal-Wallis, combinado com o post-hoc de Bonferroni. Os resultados dos testes revelaram que o fator Cooperação e Integração foi o que mais influenciou na formação dos agrupamentos, conforme os resultados do teste $\left(\chi^{2}=50,70\right.$, sig<0,01) e que, de maneira geral, os agrupamentos estavam corretamente classificados, com resultados significativos entre 1 e $10 \%$ para a maioria das variáveis (Tabela 2). 
Tabela 2 - Teste de Kruskal-Wallis com post-hoc Bonferroni

\begin{tabular}{|c|c|c|c|c|}
\hline & & Fator 1 & Fator 2 & Fator 3 \\
\hline \multicolumn{2}{|c|}{ Krushal-Wallis (qui-quadrado) } & 40,10 & 50,70 & 17,38 \\
\hline \multicolumn{2}{|l|}{ P-valor } & $<0,01$ & $<0,01$ & $<0,01$ \\
\hline \multirow{6}{*}{$\begin{array}{l}\text { Comparação } \\
\text { Múltipla }\end{array}$} & Grupo 1 vs. Grupo 2 & $<0,01$ & $<0,01$ & NS \\
\hline & Grupo 1 vs. Grupo 3 & $<0,01$ & NS & $<0,01$ \\
\hline & Grupo 1 vs. Grupo 4 & NS & $<0,01$ & $<0,01$ \\
\hline & Grupo 2 vs. Grupo 3 & NS & $<0,01$ & $<0,10$ \\
\hline & Grupo 2 vs. Grupo 4 & $<0,01$ & NS & $<0,10$ \\
\hline & Grupo 3 vs. Grupo 4 & $<0,01$ & $<0,01$ & NS \\
\hline
\end{tabular}

Legenda: NS=não significativo; Fator 1 = Imagem e Desempenho Organizacional; Fator 2 = Cooperação e Integração; Fator 3 = Carreira e Oportunidades. Fonte: Pesquisa de campo.

Quanto às características dos grupos (Figura 1), o Grupo 1 foi o menor agrupamento da pesquisa, pois reuniu apenas $12 \%$ (9) dos entrevistados. Neste grupo, os gestores avaliaram negativamente a Imagem e Desempenho Organizacional, Fator 1, que obteve escore médio de $-0,81( \pm 0,67)$, e a Cooperação e Integração com $-1,10( \pm 0,80)$, percebida como ainda mais prejudicial ao clima organizacional que a Imagem e Desempenho Organizacional. Contudo, esse grupo foi o que reuniu os entrevistados que fazem a melhor avaliação da Carreira e Oportunidades, com média de 1,10 $( \pm 0,92)$ para os escores desse fator. Destaca-se que não foram identificadas diferenças socioeconômicas entre os grupos.

O Grupo 2 destacou-se como o segundo mais numeroso da pesquisa, com 34\% (25) dos entrevistados. Ele se caracterizou pelas avaliações positivas para todos os fatores, sendo o grupo que manifestou as melhores avaliações para a Imagem e Desempenho Organizacional e para a Cooperação e Integração, ambos com escores positivos de 0,58 $( \pm 0,63)$ e $0,84(0,31)$, respectivamente. Entretanto, esse grupo percebeu fracamente as possibilidades de Carreira e Oportunidades, o que pode ser percebido pelo escore de 0,26 $( \pm 0,70)$. 
Figura 1 - Características dos agrupamentos com base nos escores fatoriais.

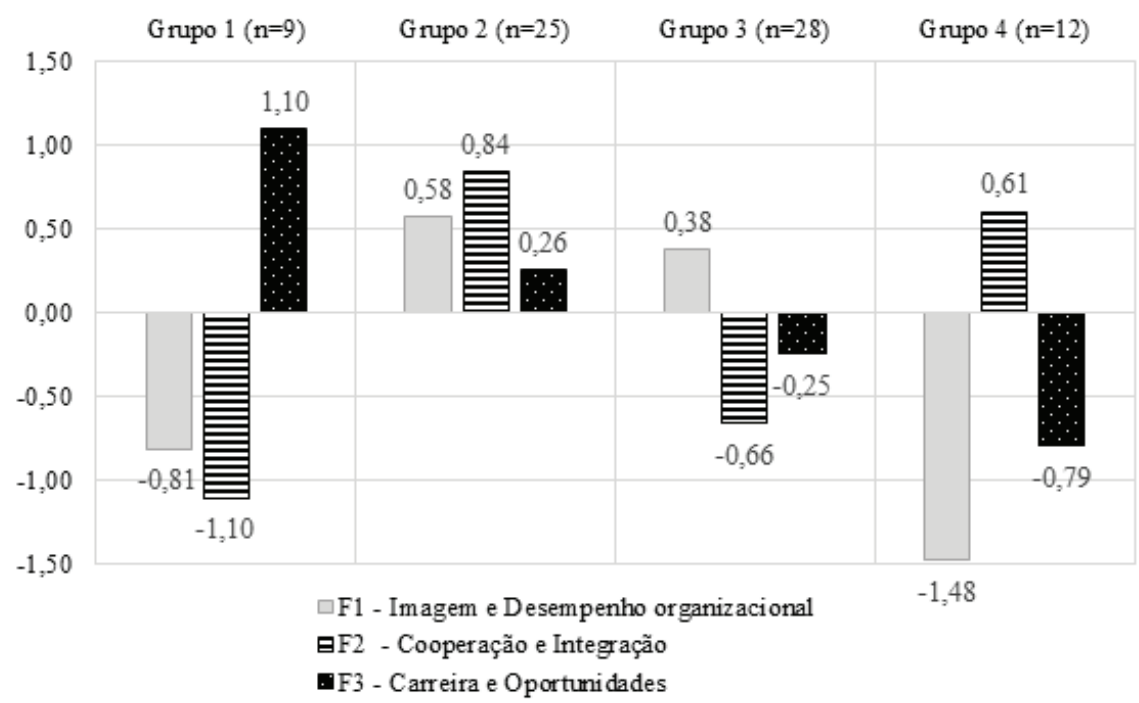

Fonte: Pesquisa de campo.

O terceiro agrupamento, Grupo 3, foi o mais numeroso da pesquisa, com 38\% (28) dos entrevistados. Esse grupo não considerou que a Cooperação e Integração e as possibilidades de Carreira e Oportunidades fossem aspectos favoráveis ao clima organizacional, uma vez que os escores foram negativos, $-0,66( \pm 0,68)$ e $-0,25( \pm 0,73)$. A única avaliação favorável nesse grupo, porém com baixa intensidade, foi a Imagem e Desempenho Organizacional, que obteve escore de 0,38 $( \pm 0,63)$.

O quarto agrupamento, Grupo 4, reuniu 16\% dos integrantes, que mostraram a pior avaliação da pesquisa para a Imagem e Desempenho Organizacional $(-1,48 \pm 0,67)$, revelando um aspecto muito prejudicial ao clima. Outra avaliação negativa coube à possibilidade de Carreira e Oportunidades, que obteve escore de $-0,79( \pm 1,30)$. Entretanto, mesmo considerando esses aspectos, o Grupo 4 percebeu como positiva a Cooperação e Integração, que foi considerada favorável ao clima organizacional, com escore médio de 0,61 $( \pm 0,84)$. 


\section{ConCluSÃo}

O objetivo do estudo foi identificar aspectos do clima organizacional, segundo a avaliação dos gestores de IFES no estado do Pará. O resultado da investigação revelou profissionais experientes, com alta escolaridade, que, de maneira geral, recomendam suas instituições como um bom lugar para trabalhar. Nas análises multivariadas, a análise fatorial, utilizada para sumarizar as variáveis e identificar variáveis latentes, apresentou boa adequação aos dados e possibilitou identificar três fatores, que explicaram a maior parte da variância dos dados. Esses fatores foram: Imagem e Desempenho Organizacional, Cooperação e integração e Carreira e Oportunidades. A consistência interna, medida pelo alpha de Cronbach, com valores elevados, revelou boa fidedignidade, tanto dos fatores, quanto das variáveis em conjunto.

Observou-se que os fatores mostraram questões típicas da atuação estratégica de competência dos gestores. Esse aspecto, em certa medida, revela que a avaliação do clima organizacional por esses profissionais assume dimensões específicas e, provavelmente, diferentes dos demais trabalhadores, uma vez que considera uma perspectiva organizacional mais ampla. Nesse sentido, os resultados mostraram porque as chefias são consideradas, em muitos modelos, como uma variável independente, mesmo considerando que esses profissionais não são imunes ao clima organizacional. Desta forma, esses achados contribuem para melhorar a compreensão do fenômeno. Assim, a preocupação com o desempenho e a cooperação interna, quando combinados com a responsabilidade em buscar os objetivos organizacionais, justificam a posição dos gestores como fortes influenciadores do clima organizacional. Com isso, o presente estudo contribui para melhorar a compreensão dos construtos que formam o clima organizacional em instituições de ensino e podem instigar outras pesquisas com objetivos semelhantes em outras regiões do país.

A análise de agrupamentos, calculada com base nos valores dos escores fatoriais, classificou os entrevistados em quatro grupos. Assim, apesar das opiniões bem diferentes entre os grupos, observou-se que $34 \%$ dos entrevistados avaliaram de forma favorável todos os fatores identificados no estudo, porém com intensidade baixa ou 
intermediária. Os demais grupos demandam mudanças, ora em um, ora em outro fator, para favorecer a criação de um clima organizacional mais satisfatório. Como sugestão, as políticas de gestão de pessoas poderiam estabelecer melhor os objetivos organizacionais e comunicá-los com maior clareza, reduzir barreiras que dificultam ações mais integradas entre os servidores e as unidades e, se ausentes, estabelecer critérios claros e imparciais para o exercício dos cargos de gestão não eletivos.

\section{REFERÊNCIAS}

ABREU, N. R.; BALDANZA, R. F.; ALMEIDA, N. H. S.; SILVA, E. S.; Comunicação e os Fatores Externos Intervenientes no Clima Organizacional em um Hospital Universitário. Qualitas Revista Eletrônica v. 14, n. 1, 2013.

ARABACI, I. B. Academic and administration personnel's perceptions of organizational climate: sample of educational faculty of Firat University. Procedia Social and Behavioral Sciences, v. 2, n. 2, p. 4445-4450, 2010.

ARAÚJO, M. C. S. Q; ABBAD, G. S.; FREITAS, T. R. Avaliação qualitativa de treinamento. Revista Psicologia: Organizações e Trabalho. Brasília, v. 17, n. 3, p. 171-179, jul. / set. 2017.

ASSIS, L. O. M.; REIS NETO, M. T. Remuneração variável no setor público: investigação das causas do fracasso e implicações para o Estado brasileiro. Revista Eletrônica de Gestão Organizacional. N. 3, v. 9, p. 585-614, set. /dez. 2011.

BAHRAMI, M. A.; BARATI, O.; GHOROGHCHIAN, M., MONTAZER-ALFARAJ, R,; EZZATABADI, M. R. Role of organizational climate in organizational commitment: the case of teaching hospitals. Osong Public Health Res Perspect, n. 7, v. 2, p. 96-100, apr. 2016.

BARBOSA, F. L. S.; BIZARRIA, F. P. A.; RABÊLO NETO, A.; MOREIRA, R. N. Visão multidimensional da satisfação do trabalho: um estudo em um hospital público piauiense. REGE, São Paulo, v. 23, n. 2, p. 99-110, abr. / jun. 2016.

BENDASSOLLI, P. F. Reconhecimento no trabalho: perspectivas e questões contemporâneas. Psicologia em Estudo, Maringá, v. 17, n. 1, p. 37-46, jan. / mar. 2017.

BERGUE, S.T. Modelos de gestão em organizações públicas. Caxias do Sul: Educs, 2011.

BISPO, C. R. F. Um novo modelo de pesquisa de clima organizacional. Produção, v. 16, n. 2, p. 258-273, maio/ago. 2006.

BRANDÃO, H. P.; BORGES-ANDRADE, J.E.; TOMÁS, A. G. Desempenho organizacional e suas relações com competências gerenciais, suporte organizacional e treinamento. Revista de Administração, v. 47, n. 4, p. 523-539, out./dez., 2012

BRAND-LABUSCHAGNE, L.; MOSTERT, K.; ROTHMANN JNR; ROTHMANN, C. Burnout and engagement of South African blue-collar workers: the development of a new scale. African Journals Online, v. 16, n. 1, p. 58-93, 2012. 
CAMPOS, K. C. L. Análise do clima organizacional do curso de psicologia de uma universidade comunitária. Psicologia Escolar e Educacional, n. 2, v. 6, p. 123-131, dez. 2002.

CANTOS, G. A.; SILVA, M. R.; NUNES, S. R. L. Estresse e seu reflexo na saúde do professor. Saúde em Revista, n. 7, v. 15, p. 15-20, 2005.

CAVAZOTTE, F.S.C.N.; MORENO JR, V. A.; TURANO, L. M. Cultura de aprendizagem contínua, atitudes e desempenho no trabalho: uma comparação entre empresas do setor público e privado. Revista de Administração Pública, n. 49, v. 6, p. 1555-1578, nov./dez. 2015.

CODA. Roberto. Pesquisa de clima organizacional e gestão estratégica de recursos humanos. In: BERGAMINI, Cecília W.; CODA, Roberto. Psicodinâmica da vida organizacional: motivação e liderança. São Paulo: Atlas, 1997.

COSTA, F. J. Mensuração e desenvolvimento de escalas. Rio de Janeiro: Ciência Moderna, 2011.

D’ALLEO, G.; SANTANGELO, A. Organizational Climate and Burnout in Call-Center Operators. Procedia, n. 30, p. $1608-1615,2011$.

DILLON, W. R.; GOLDSTEIN, M. Multivariate analysis: methods and applications. New York: John Wiley \& Sons, 1984.

FÁVERO, L. P.; BELFIORE, P.; SILVA, F. L.; CHAN, B. L. Análise de dados: modelagem multivariada para a tomada de decisão. Rio de Janeiro: Elsevier, 2009.

FOREHAND, G. A; GILMER, B. H. Environmental variation in studies of organizational behavior, Psychological Bulletin, v. 62, n. 6, p. 361-382, 1964.

FRANÇA, J. C. A. F.; MATTOS, C. A. C. Clima organizacional e administração pública municipal: uma investigação na cidade de Belém do Pará, Brasil. Revista Gestão em Análise, Fortaleza, v. 6, n. 1/2, p. $114-130$, jan./ dez. 2017.

FRANCHI, T. S., ARENHARDT, L. D. A percepção do clima organizacional no serviço público: um estudo com servidores técnico-administrativos da Universidade Federal de Santa Maria. Observatorio de la Economía Latinoamericana, n. 201, 2014.

GRIFFITH, J. A compositional analysis of the organizational climate-performance relation: public schools as organizations. Journal of Applied Social Psychology, v.35, n.8, p. 18401880, agu. 2006.

HAIR, J. F. Jr; BLACK, W. C.; BABIN, J.; ANDRESON, R. E.; TATHAM, R. L. Análise multivariada de dados. São Paulo: Bookman, 2009.

HOLLOWAY, J. B. Leadership Behavior and organizational climate: an empirical study in a non-profit organizational. Energing Leandership Journeys, v.5, n. 1, p. 9 -35. 2012.

HORA, G.P.R.; RIBAS JÚNIOR, R. SOUZA, M. A. Estado da arte das medidas em satisfação no trabalho: uma revisão sistemática. Temas em Psicologia, Ribeirão Preto, v. 26, n. 2, p. 971-986, abri. / jun. 2018.

HU, X.; KAPLAN, S.; DALAL, R.S. An examination of blue versus white-collar workers' conceptualizations of job satisfaction facets. Journal of Vocational Behavior, n. 2, v. 76, p. 317-325, apr. 2010 
JAHANI, M. A.; YAMINFIROOZ, M.; SIAMIAN, H. The regression model of Iran libraries organizational Climate. Mater Sociomed, n. 27, v. 5, p. 342-346, oct. 2017.

JING, F. F.; AVERY, G.C.; BERSTEINEIR, H. Organizational climate and performance in retail pharmacies. Leadership \& Organization Development Journal, n. 3, v. 32, p. 224-242, 2011.

KJELDSEN, A.M.; HANSEN, J.R. Sector differences in the public service motivation- job satisfaction relationship: exploring the role organizational characteristics. Review of Public Personnel Administration, v. 38, n. 1, p. 24-48, fev. 2016.

KOLB, D. A; Rubin, I.; Mclntyre, J. Organizational psychology: an experimental approach. New Jersey: Prentice Hall, 1978.

KOOTER, J.P.; HESKETT, J. L. Corporate culture and performance. New York, 2011.

KUBO, E. K. M. et al. Frankenstein e o clima organizacional: estudo bibliográfico sobre um construto indefinido. Revista Internacional de Investigación en Ciencias Sociales, v. 11, n. 1, p. 35-54, jan. /jul., 2015.

LADEO, C. M.; SPIER, K. E. Incentive contracts of teams: experimental evidence. Journal of Economic Behavior \& Organization, v. 119, p. 496-511, nov. 2015.

LANER, A. S.; CRUZ JÚNIOR, J. B. Repensando as organizações - da formação à participação. Florianópolis: Fundação Boiteux, p. 317-338, 2004.

LIM, S.; EO, S. The mediating roles of collective teacher efficacy in the relations of teachers' perceptions of school organizational climate to their burnout. Teaching and Teacher Education. v. 44, p. 138 - 147, nov. 2014.

LITWIN, G.H; STRINGER, R.A. Motivation and organizational climate. Cambridge: Harvard University Press, 1968.

LU, C.; CHEN, S.; HUANG, P.; CHIEN, J. Effect of diversity on human resource management and organizational performance. Journal of Business Research, n. 68, v. 4, p. 857-861, apr. 2015.

LUCHESI, J. R. De S.; CRESPI, K. M., CAMARGO, M. E. Pesquisa de clima organizacional: metodologia proposta para uma instituição de ensino superior da Serra Gaúcha. Qualitas Revista Eletrônica, n. 1, v. 1, jan. / jun. 2011

LUZ, R. Clima Organizacional. Rio de Janeiro: Qualitymark, 2012.

MAJEED, A. SHAKEEL, S. Importance of training and development in the workplace. International Journal of Scientific \& Engineering Research, v. 8, n. 1, p. 498-504, apr. 2017.

MARTELO, M. R.; CLARO, J. A. C. S.; VIEIRA, A. M. Clima Organizacional No Contexto De Uma Incubadora: Estudo Com Três Empreendimentos Do Interior De São Paulo. Revista FSA (Faculdade Santo Agostinho), v. 13, p. 24-49, 2016.

MARTINS, G. A.; THEÓPHILO, C. R. Metodologia da investigação científica para ciências para Ciências Sociais Aplicadas. São Paulo: Atlas, 2017.

MATTOS, C.A.C.; DAMASCENO, V. B.; GAMA, M. F. C.; SIMÃO, C. S., COSTA, N. L. Qualidade de vida no trabalho: uma investigação multivariada entre docentes. Revista da Raunp, Natal, v. 10, n. 1, p. 77-91, jun./nov. 2017. 
MAXIMILIANO, A. C. A., Teoria Geral da Administração. São Paulo: Atlas, 2012.

MEJÍAS, A. Agustín; REYES S. Oscar; ARZOLA, H. Minerva. Medición del clima organizacional en instituciones de educación superior. Universidad, Ciência y Tecnonologia, v. 30, n. 38 , p. 55- 61, jun. 2006.

MINGOTI, S. A. Análise de dados através de métodos de estatística multivariada: uma abordagem aplicada. Belo Horizonte: Editora UFMG, 2007.

MÓL, A. L. R.; FERNANDES, A.S.; TINÔCO, D. S.; BORGES, D. F.; ALLOUFA, J. M. L.; ARAÚJO, M. A. D. Clima organizacional na administração pública. Rio de Janeiro: FGV Editora, 2010.

MORAN, E. T.; VOLKWEIN, J. F. The cultural approach to the formation of organizational climate. Human Relations, n. 1, v. 45, p. 19-47, 1992.

MORO, A. B. et. al. Avaliação do Clima Organizacional dos Servidores Técnico- Administrativos de uma Instituição Pública de Ensino. In: ENAPG - Encontro de Administração Pública e Governo, 2012, Salvador. Anais... Salvador: ANPAD - Associação Nacional de Pós-graduação e Pesquisa em Administração, 2012.

OCDE Organization for Economic Co-operation and Development. Perfomance-related pay policies for government employees. Paris: OCDE Publishing, 2005.

PATTERSON M. G., WEST M. A., SHACKLETON V. J., DAWSON J. F., LAWTHOM, R., MAITLIS S., ROBINSON, D. L., WALLACE, A. M. Validating the organizational climate measure: links to managerial practices, productivity and innovation. Journal of Organizational Behavior, n. 26 p. 379-408, apr. 2005.

PEREIRA, J. M.; VIDAL, M.; A Influência do Clima Organizacional no Desempenho das Pessoas: Um Estudo de Caso no Fórum da Comarca de Bezerros. Veredas Favip Revista Eletrônica de Ciências, n. 1, v. 4, p. 123-138, jan. / jun. 2011.

RIZZATTI, G. Categorias de análise de clima organizacional em universidades federais brasileiras. Florianópolis, 2001. Tese. (Doutorado em Engenharia de Produção) - Universidade Federal de Santa Catarina, Programa de Pós-Graduação em Engenharia de Produção, Florianópolis, 2002.

RUSU, G.; AVASILCAI, S. Linking human resources motivation to organizational climate. Procedia - Social and Behaviorial Sciences, n. 124, p. 51-58, mar. 2014.

SALGADO NETO, J. B. Clima organizacional da UEMA: uma contribuição ao Programa “Uma Universidade para o Maranhão". Florianópolis, 2001. Dissertação (Mestrado em Administração) - Universidade Federal de Santa Catarina, Programa de Pós-Graduação em Administração, Florianópolis, 2001.

SALGADO, C. C. R.; AIRES, R. F. F.; ARAÚJO, A. G. Clima Organizacional: Um Estudo de uma Superintendência Estadual de Um Banco Múltiplo. Tekhne e Logos, n. 1, v. 4, p. 50 64, ago, 2013.

SANTOS, L. C.; VÁSQUEZ, O. C. A Pesquisa de clima organizacional como instrumento de suporte à avaliação nas instituições de ensino superior. Avaliação, v. 17 n.1, p. 43-63, mar. 2012.

SCHAUFELI; W.; DIJKSTRA, P.; VAZQUEZ, A. C. Engajamento no trabalho. São Paulo: 
Casa do Psicólogo, 2013.

SCHNEIDER, B. Organizational climates: an essay. Personnel Psychology, n. 28, p. 447-479. dec. 1975.

SHANKER, R.; BHANUGOPAN, R.; VAN DER HEIJDEN, B, I. J. M.; FARRELL, M. Organizational climate for innovation and organizational performance: the mediating effect of innovative work behavior. Journal of Vocational Behavior, n. 100, p. 67-77, jun. 2017.

SHIM, M. Factors influencing child welfare employee's turnover: focusing on organizational culture and climate. Children and Youth Services Review, n. 32, p. 847 - 856, jun. 2010.

SILVESTRE, H. C. Gestão pública: modelos de prestação no serviço público. Lisboa: Escolar, 2010.

SORATTO, J.; PIRES, D. E. P.; TRINDADE, L. L.; OLIVEIRA, J. S. A.; FORTE, E. C. N.; MELO, T. P. Insatisfação no trabalho de profissionais da saúde na estratégia saúde da família. Texto e Contexto em Enfermagem, Florianópolis, v. 26, n. 3, p. 1-11, out. / dez. 2017.

STRINGER, R. A. Leadership and organizational climate: the cloud chamber effect. New Jersey: Prentice Hall, Upper saddler, 2002.

TACHIZAWA, T. e ANDRADE, R. O. Gestão de instituição de ensino. Rio de Janeiro: FGV Editora, 2008.

TONGO, C. I. Social responsibility, quality of work life and motivation to contribute in the Nigerian Society. Journal os Business Ethics, v. 126, n. 2, p. 219-233, jan. 2015.

VERGARA, S. C. Projetos e relatórios de pesquisa em administração. São Paulo: Atlas, 2014.

VIEIRA, A. M. Cultura organizacional em instituições de ensino: mapeamento e análise descritivo-interpretativa da produção acadêmica (1990-2005). 2007. 237f. Tese (Doutorado em Educação) - Universidade Estadual Paulista (UNESP), Marília, 2007.

Recebido em: 25-9-2017

Aprovado em: 1-6-2019

Avaliado pelo sistema double blind review.

Editor: Coordenação do PPGA/UMESP

Disponível em http://mjs.metodista.br/index.php/roc 